\title{
LPS Pretreatment Attenuates Cerebral Ischaemia/Reperfusion Injury by Inhibiting Inflammation and Apoptosis
}

\author{
Zhiquan Lva Chong Liub Meili Zhaic ${ }^{\mathrm{b}}$ Qian Zhang ${ }^{\mathrm{c}} \quad$ Jun Lid ${ }^{\mathrm{d}} \quad$ Fang Zheng $^{\mathrm{e}}$ \\ Mingqing Peng ${ }^{\mathrm{a}}$ \\ aDepartment of Anaesthesiology, Yongchuan Hospital, Chongqing Medical University, Chongqing, \\ ${ }^{b}$ Department of Anaesthesiology, Tianjin Fourth Central Hospital, The Fourth Central Hospital \\ Affiliated to Nankai University, Tianjin, 'Department of Anaesthesiology, Tianjin Obstetrics Centre and \\ Gynaecology Hospital, Central Obstetrics and Gynaecology Hospital of Nankai University, Tianjin, \\ dDepartment of Anaesthesiology, People's Hospital of Xuyong County, Xuyong, eSchool of Integrative \\ Medicine, Tianjin Traditional Medical University, Tianjin, China
}

\section{Key Words}

Lps pretreatment $\cdot$ Cerebral ischaemia/reperfusion injury Inflammation • Apoptosis

\begin{abstract}
Background/Aims: Lipopolysaccharide (LPS) pretreatment has a strong neuroprotective effect on cerebral ischaemia/reperfusion injury (IRI), but the mechanism has not been fully elucidated to date. This study investigated the effect of LPS pretreatment on the pathway mediated by endoplasmic reticulum (ER) stress-CCAAT/enhancer-binding protein- homologous protein (CHOP) and the role of this pathway on cerebral ischaemia/reperfusion (I/R)-induced inflammation and apoptosis. Methods: Healthy male BALB/c mice were randomised into four groups as follows: sham operation group (sham group, $n=30$ ); LPS group (BALB/c mice treated with LPS, $n=30$ ); ischaemia/reperfusion group (I/R group, $n=30$ ) and $I / R+L P S$ group (BALB/C mice treated with LPS before ischaemia, $n=30)$. The mice were pre-treated with LPS $(0.2 \mathrm{mg} / \mathrm{kg})$ intra-peritoneally for three days prior to cerebral ischaemia. After 24 hours, the neurological deficit score, TTC staining and TUNEL assay were used to assess the neuroprotective effect of the LPS pretreatment against cerebral IRI. To assess whether the ER stress-CHOP pathway participated in the LPS-pretreatment neuroprotective mechanism, the expression levels of related proteins (GRP78, CHOP, caspase-12 and caspase-3) from the ischaemic cortical penumbra were detected via a western blot analysis. An immunohistochemical study was used to detect the expression and location of $\mathrm{CHOP}$ in the cortical penumbra. To further assess the protective effect of the LPS pretreatment, the concentrations of inflammatory factors (TNF- $\alpha$, IL-6, IL-1 $\beta$ and IL-10) in the cortical penumbra were measured by ELISA, and ER stress-CHOP pathway inflammation-related caspase- 11 was analysed through western blot analysis. Results: As demonstrated by the experiments, the pretreatment with LPS significantly reduced the

Z. LV, and C. Liu contributed equally to this publication; Co- corresponding author M. Peng and F. Zheng contributed equally to this work.
\end{abstract}

Mingqing Peng, and Fang Zheng

\section{KARGER}

Dpt. Anaesthesiol, Yongchuan Hospital, Chongqing Medical University; Dpt. of Anaesthesiol, School of Integrative Medicine, Tianjin Traditional Medical University, (China); E-Mail pengmingqing88@163.com, zhengfang_1979@126.com 
neurological deficit score and the infarct size of cerebral IRI. The expression levels of ER stressCHOP pathway related proteins (GRP78, CHOP, caspase-12 and caspase-3) from the cortical penumbra were significantly decreased by LPS, as well as the level of apoptosis in the cells in the brain. Immunohistochemistry showed that the expression of CHOP significantly decreased after the LPS pretreatment. Furthermore, the concentrations of inflammatory factors (TNF- $\alpha$, IL-1 $\beta$, IL-6) were reduced after the LPS pretreatment, whereas the anti-inflammatory cytokine IL-10 was upregulated. In addition, ER stress-CHOP pathway inflammation-related caspase-11 expression was significantly suppressed after the pretreatment with LPS. Conclusions: LPS pretreatment significantly ameliorates the effects of cerebral IRI by inhibiting inflammation and apoptosis, and the potential mechanism of the neuroprotective effect may be associated with the ER stress-CHOP mediated signalling pathway.

(C) 2018 The Author(s)

Published by S. Karger AG, Basel

\section{Introduction}

Cerebral ischaemia/reperfusion injury (IRI) leads to mortality and disability, causing a serious socioeconomic burden. The ischaemic centre of the brain tissue, which is caused by a transient middle cerebral artery occlusion, has a severe characteristic pathophysiological change, and ischaemic penumbra plays a key role in cerebral IRI. The ischaemic penumbra is defined as the region around necrosis after cerebral ischaemia with a lower level of blood perfusion than normal brain function but higher than the ischaemic centre area with a changed morphological structure. The ischaemic penumbra progressively deteriorates within several days of stroke [1]. However, when the blood flow is restored, its function may be reversed, at least partially. Therefore, the rescue of ischaemic penumbra neurons is a key treatment strategy for acute cerebral IRI [2]. Timely and successful cerebral reperfusion is the most effective method to reduce the infarct size and morbidity [3]. Cerebral IRI involves an extremely complex pathophysiological process, such as calcium-homeostasis disorder, oxidative stress and endoplasmic reticulum (ER) dysfunction, eventually leading to cell necrosis or apoptosis [4]. However, the precise mechanism has not been thoroughly elucidated to date. ER stress plays an important role in cerebral damage after ischaemia/ reperfusion (I/R) [5]. Mild tissue I/R leads to the disturbance of ER function, resulting in an unfolded protein response, which relieves the stress of the cells. Nevertheless, persistent and severe stress, which exceeds the ability of the ER to address the unfolded protein response, ultimately triggers the ER stress apoptotic pathway [6]. The protein level of ER chaperone glucose-regulated protein 78/immunoglobulin heavy chain binding protein (Grp78/BiP), which is mainly present in the ER and is used as a whistle marker for ER stress, is increased under pathological conditions, such as Alzheimer's disease, Parkinson's disease and cerebral IRI [7]. Meanwhile, the ER stress response pathway is triggered, which is mediated by the activation of three endoplasmic transmembrane proteins, including Ire1 (inositol requiring enzyme 1), PERK (pancreatic endoplasmic reticulum kinase) and ATF6 (activating transcription factor 6) [8]. When the ER function is severely impaired, the impaired cells are removed through the apoptotic mechanism. Apoptosis is caused by the inclusion of C/EBP homologous protein (CHOP)/ DNA damage-inducible gene 153 (GADD153), apoptosis signalregulating kinase 1 (ASK1) and caspase-12 [9-12]. Induction of CHOP, which is a transcription factor that belongs to the C/EBP transcription factor family, is a signalling event underlying ER stress-induced apoptosis. It is confirmed that CHOP-induced apoptosis is involved in the pathogenesis of many diseases, including cancer, diabetes, fibrosis, neurodegenerative disease and brain ischaemia $[9,13]$.

Lipopolysaccharide (LPS), known as the antigenic component of the gram-negative bacterial cell wall, may initiate the activation of Toll-like receptor 4 (TLR4), causing severe sepsis and inflammation [14]. Recently, the literature reports that the ER stress-CHOP pathway participates in the pathogenesis of LPS-induced lung inflammation [15], liver IRI [16], and pancreatitis [17]. Several articles have further shown that the ER stress-CHOP pathway plays a key role in the pathogenesis of LPS-induced inflammation by inducing 


\section{Cellular Physiology Cell Physiol Biochem 2018;45:2246-2256

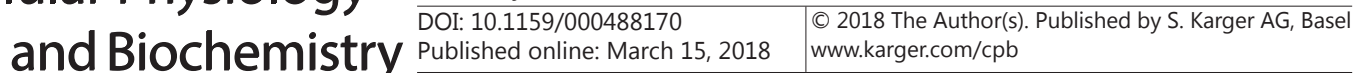

Ly et al.: LPS Pretreatment and Cerebral Ischaemia/Reperfusion Injury

caspase-11 [15-19]. Caspase-11 is called an inflammation-related caspase because the active form of caspase- 11 proteolysis converts procaspase- 1 to caspase- 1 , which activates pro-IL$1 \beta$ to IL-1 $\beta$, eventually leading to inflammation $[18,20]$. Therefore, we know that the ER stress-CHOP pathway is highly important in the pathogenesis of many organ inflammation responses. Although it is documented that a low dose LPS pretreatment protects the liver, brain, heart and intestine from IRI by inhibiting the inflammatory response [16, 21-23], the potential neuroprotective effect on brain cell inflammation and apoptosis after cerebral IRI is not completely elucidated. The target of our research aimed to explore the influence of LPS pretreatment on the pathway mediated by ER stress-CHOP and the role of this pathway on cerebral IRI-induced inflammation and apoptosis.

\section{Materials and Methods}

\section{Animals}

Healthy male (clean grade) BALB/c mice (8-10 weeks old, 25-30 g) were obtained from the Experimental Animal Centre of Chongqing Medical University (Chongqing, China). In our study, all of the experiments were conducted according to the Guidelines of the Ethics Committee for Laboratory Animals in Chongqing Medical University. All of the mice were kept in a laboratory room at $22-25^{\circ} \mathrm{C}$ temperature with a 12-hour light-dark cycle and had free access to food and water.

\section{Surgery and experimental design}

The focal cerebral ischaemia model was replicated by middle cerebral artery occlusion (MCAO) as previously described [24]. Briefly, the rats were anaesthetised with $10 \%$ chloral hydrate $(300 \mathrm{mg} / \mathrm{kg}$ ) intraperitoneally, and the middle cerebral artery was later blocked for 2 hours and was reperfused for 24 hours.

The mice were randomly divided into four groups as follows: sham operation group (sham group, $\mathrm{n}=30$ ); LPS (Escherichia coli 0111:B4, Sigma Aldrich, USA) group (LPS group, $n=30$ ); cerebral ischaemia/ reperfusion group (I/R) group (I/R group, $n=30$ ) and $I / R+L P S$ group (I/R+LPS group, $n=30$ ). The sham operation mice followed the same procedure as the other groups, except for the arterial occlusion.

The mice in the LPS and I/R+LPS groups were injected by an intra-peritoneal administration with 0.2 $\mathrm{mg} / \mathrm{kg}$ LPS for three consecutive days before the I/R operation. However, in the sham and I/R groups, an equal volume of saline was administered for three consecutive days prior to I/R. The neurological deficit score was measured after 24 hours of reperfusion. Subsequently, each rat was sacrificed, and then, the brain cell apoptosis, the concentration of the inflammatory factors and the ER stress-CHOP apoptotic pathwayrelated protein expressions were measured.

Neurological deficit score assessment

Twenty-four hours after reperfusion, a neurological behavioural test was performed for each rat according to Longa and Weinstein's method [24], and the data were obtained from an experimenter who was unaware of the experimental design.

\section{Infarct volume assessment}

The animals were sacrificed following the neurological function assessment. The brains were rapidly removed and cut into $2 \mathrm{~mm}$ thick coronal pieces and submerged in a 2\% solution of 2, 3,5-triphenyltetrazole (TTC, Sigma) staining at $37^{\circ} \mathrm{C}$ for $20 \mathrm{~min}$. The brain slices were subsequently fixed overnight in $4 \%$ paraformaldehyde at $4^{\circ} \mathrm{C}$. The infarcted area was white, and the non-infarct tissue was red. The size of the infarct (white) from each site was analysed using image analysis software (image-Pro Plus 6.0). The degree of the infarct area was assessed by the following formula: (contralateral hemisphere volume - non-infarct ipsilateral hemisphere volume) / contralateral hemisphere volume $\times 100 \%$ [25].

The ipsilateral hemisphere region, corresponding to the ischaemic core and penumbra, was identified by TTC staining. The middle coronal brain slice was used to determine the ischaemic penumbra [26]. We initially cut approximately $2 \mathrm{~mm}$ longitudinally (from top to bottom) through the right hemisphere from the lateral side of the sagittal suture. A transverse diagonal cut was then performed at approximately the "2 


\section{Cellular Physiology Cell Physiol Biochem 2018;45:2246-2256 \\ \begin{tabular}{ll|l} 
DOI: 10.1159/000488170 & and Biochemistry Published onlne: TVarch 15, 2018 & $\begin{array}{l}\text { O 2018 The Author(s). Published by S. Karger AG, Basel } \\
\text { www.karger.com/cpb }\end{array}$
\end{tabular}}

Ly et al.: LPS Pretreatment and Cerebral Ischaemia/Reperfusion Injury

o'clock" position, and eventually, the penumbra was separated from the core for further experiments [27]. However, based on previous studies, we made subtle changes to the ischaemic core and penumbra [28-31]. The infarction area was located in the lateral caudoputamen and the adjacent ventrolateral cortex of the frontal parietal, while the penumbra area was located in the medial caudoputamen and adjacent to the dorsal medial cortex of the frontal parietal (Fig. 1C).

\section{TUNEL staining for apoptosis assessment}

The cortical penumbra of the brain tissue was embedded in paraffin and was then cut into $5 \mu \mathrm{m}$ sections for the TUNEL assay. TUNEL staining was performed in strict accordance with the manufacturer's instructions for in situ cell death detection kit (Promega, USA). The number of TUNEL-positive cells from ten randomly selected fields of the cortical penumbra in each slice was counted under an optical microscope.

\section{Enzyme-linked immunosorbent assay (ELISA)}

The concentrations of TNF- $\alpha$, IL-1 $\beta$, IL- 6 and IL-10 in the cortical penumbra supernatant were measured by mouse ELISA kits (R \& D Systems) according to the manufacturer's instructions. The experiments were carefully repeated three times to verify the results.

\section{Western blot analysis}

The cortical penumbra of the brain tissue sample was taken from the experimental mice according to our previously described method and was later quickly stored at $-80^{\circ} \mathrm{C}$ for further use. The cortical penumbra was homogenised with a glass homogeniser in ice-cold radioimmunoprecipitation assay lysis buffer (50 mM Tris-HCl, $150 \mathrm{mM} \mathrm{NaCl}$, 1\% Triton X-100, 1\% sodium deoxycholate, pH 7.4). After 30 min of incubation, the tissue homogenate was centrifuged at $800 \times \mathrm{g}$ for $5 \mathrm{~min}$ at $4^{\circ} \mathrm{C}$, and the supernatant was further centrifuged at $12,000 \times \mathrm{g}$ for $30 \mathrm{~min}$ at $4^{\circ} \mathrm{C}$. The new supernatant was applied to $12 \%$ sodium dodecyl sulfate polyacrylamide gel electrophoresis (SDS-PAGE), and the separated proteins were transferred to a polyvinylidene difluoride membrane (Millipore, Bedford, MA, USA). The membranes were blocked for 45 minutes at $4^{\circ} \mathrm{C}$ with $5 \%$ non-fat milk and were later incubated overnight at the same temperature with primary antibodies as follows: CHOP (1:500) (Santa Cruz Biotechnology); GRP78 (1:500) (Santa Cruz Biotechnology); caspase-12 (1:1000) (Bios Biotechnology Inc, China); Caspase-11 (1:1000) (Cell Signaling Technology, Danvers, MA); Caspase-3 (1:1000) (Cell Signaling Technology, Danvers, MA), $\beta$-actin(1:1000) and GAPDH (1:1000) (Santa Cruz Biotechnology, Santa Cruz, CA). After three washes with PBS-T, the membranes were incubated with peroxidase-conjugated secondary antibody (1:1000) (Santa Cruz Biotechnology, Santa Cruz, CA) at room temperature for 1 hour. The protein bands were quantified by use of Quantity one software (Bio-Rad, Hercules, CA, USA). GAPDH and $\beta$-actin were chosen as the internal controls. All of the band intensities were normalised to GAPDH or $\beta$-actin and are expressed as a percentage of the control samples.

\section{Immunohistochemical study}

An immunohistochemical study was used to detect the expression of CHOP in the cortical penumbra according to the manufacturer's instructions. Briefly, the paraffin embedded brain sections were dewaxed and washed three times in PBS and were later immersed in 3\% hydrogen peroxide for 3 minutes to block endogenous peroxidase and quenched for 20 minutes in BSA. Next, the sections were incubated with a rabbit polyclonal antibody against $\mathrm{CHOP}$ (1: 200 dilution) at $4{ }^{\circ} \mathrm{C}$. Next, the sections were incubated with an anti-rabbit secondary antibody for 1 hour at $4^{\circ} \mathrm{C}$. After that, 3, 3'-diaminobenzidine (DAB) was used for colour development. Finally, haematoxylin was used for counterstaining. The number of apoptotic cells in each area was calculated. Random high-power fields from five different cortical penumbra in each section were examined. A high magnification $(400 \times)$ photograph was taken using a light microscope. The CHOP positive cell ratios were analysed using Image-Pro Plus 6.0 software.

\section{Statistical analysis}

All the data are shown as the mean \pm SD. All the statistical analyses were performed with SPSS version 19.0 software. Comparisons among multiple groups were performed with a one-way ANOVA. When the differences were considered statistically significant, the least significant difference test was performed to compare the two groups. The statistical significance was determined to be $\mathrm{P}<0.05$. 


\section{Results}

LPS pretreatment attenuates cerebral damage following IRI

Cerebral I/R models were successfully established. To determine the neuroprotective effect of the LPS pretreatment against cerebral IRI, we assessed the neurological deficit score after 24 hours of reperfusion (Fig. 1A). The neurological deficit score of the I/R group was obviously higher than the sham group $(\mathrm{P}<0.05)$. However, the neurological deficit score of the I/R+LPS group showed a significant improvement compared to the $\mathrm{I} / \mathrm{R}$ group $(\mathrm{P}<0.05)$. In addition, as demonstrated by the TTC-stained coronal sections, the infract size of the I/R group was significantly increased compared to the sham group, while the infarct size of the brain injury was also remarkably reduced in the I/R+LPS group compared with the I/R group (Fig. 1B, D, $\mathrm{P}<0.05$ ). There was no significant difference between the sham group and LPS group. Based on these results, the low dose LPS pretreatment attenuated cerebral damage following IRI, and therefore, the optimal treatment dose of LPS $(0.2 \mathrm{mg} / \mathrm{kg})$ was used in the following experiment.

\section{LPS pretreatment attenuates} cerebral apoptosis following IRI

To study the brain cell apoptosis rate of the cortical penumbra after cerebral IRI and the LPS pretreatment anti-apoptotic effect, we conducted the TUNEL assay. Compared to the control group, the rate of TUNEL-positive cells in the $I / R$ group was considerably increased ( $54.3 \pm 1.1 \%$ vs. $17.5 \pm 0.7 \%$, P $<0.05$ ). However, the rate of TUNELpositive cells in the I/R+LPS group was significantly decreased compared to the I/R group $(39.6 \pm 0.8 \%$ vs. $54.3 \pm 1.1 \%$, P $<0.05$ ). The results showed that LPS pretreatment markedly inhibited brain cell apoptosis (Fig. 2A, B).

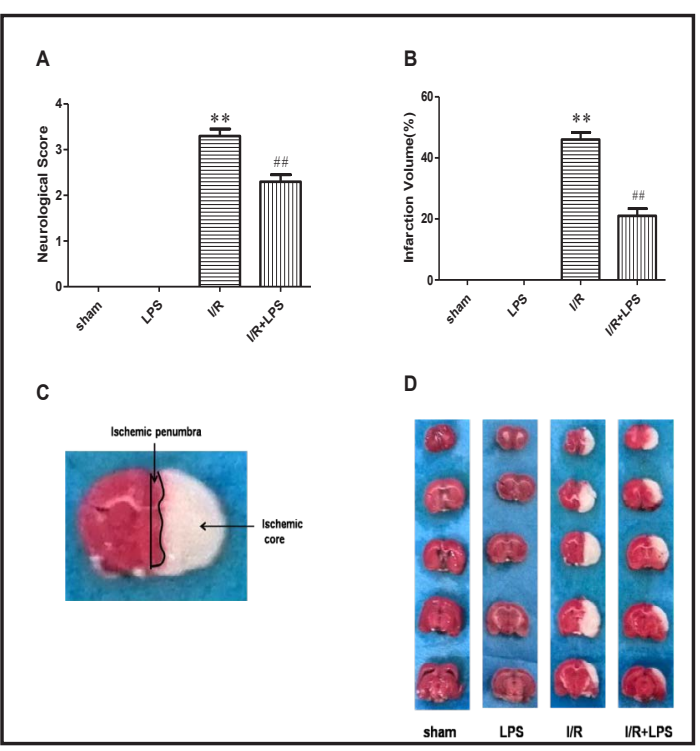

Fig. 1. Effects of low-dose LPS $(0.2 \mathrm{mg} / \mathrm{kg})$ pretreatment on neurological deficit scores and infarct volumes in the mice subjected to $2 \mathrm{~h}$ MCAO followed by $24 \mathrm{~h}$ reperfusion. A. Neurological deficit score. $\mathrm{n}=$ 30. B. Infarct size detected by TTC staining. C. The red zone near the infarction zone represented the ischemic penumbra in the ipsilateral hemisphere of coronal brain section. D. Representative TTC-stained coronal brain sections. $\mathrm{n}=6$. Data are shown as mean \pm SEM. ${ }^{*} \mathrm{P}<0.05,{ }^{* *} \mathrm{P}<0.01$ versus sham group; ${ }^{\#} \mathrm{P}<0.05,{ }^{\# \#} \mathrm{P}<0.01$ versus $\mathrm{I} / \mathrm{R}$ group.

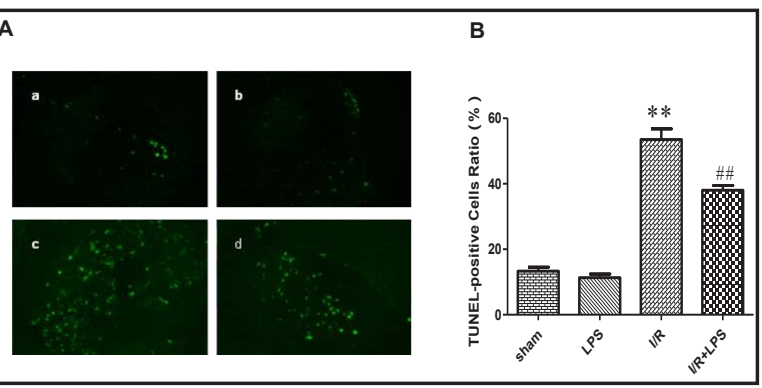

Fig. 2. Effects of low-dose LPS pretreatment on cell apoptosis in the mice subjected to $2 \mathrm{~h}$ MCAO followed by $24 \mathrm{~h}$ reperfusion. A. TUNEL staining was performed on the slices from cortical penumbra. a. sham group, b. LPS group, c. I/R group, and d. I/R + LPS group. B. Ratio of positive cells. Magnification is $200 \times . \mathrm{n}=6$. Data are expressed as mean $\pm \mathrm{SEM}$. ${ }^{*} \mathrm{P}<0.05,{ }^{* *} \mathrm{P}<0.01$ versus sham group; ${ }^{\mathrm{P}<0.05},{ }^{\# \#} \mathrm{P}<0.01$ versus I/R group.

\section{LPS pretreatment inhibits cerebral inflammation following IRI}

To further assess the protective effect of the LPS pretreatment on the brain, the expression levels of inflammatory factors (TNF- $\alpha$, IL- 6 and IL-10) from the cortical penumbra were measured by ELISA (Fig. 3). The results demonstrated that the concentrations of proinflammatory factors (TNF- $\alpha$, IL-6) in the I/R+LPS group significantly decreased compared 
Fig. 3. Effects of low-dose LPS pretreatment on inflammatory factors in the mice subjected to $2 \mathrm{~h}$ MCAO followed by $24 \mathrm{~h}$ reperfusion. Serum concentrations of inflammatoy cytokines (TNF- $\alpha$, IL-6, IL-1 $\beta$, and IL-10) from cortical penumbra were measured before and after I/R injury in four experimental groups ( $\mathrm{n}=6$ in each group). Data are expressed as mean \pm SEM. ${ }^{*} \mathrm{P}<0.05,{ }^{* *} \mathrm{P}<0.01$ versus sham group; ${ }^{*} \mathrm{P}<0.05$, \#\# $\mathrm{P}<0.01$ versus I/R group.

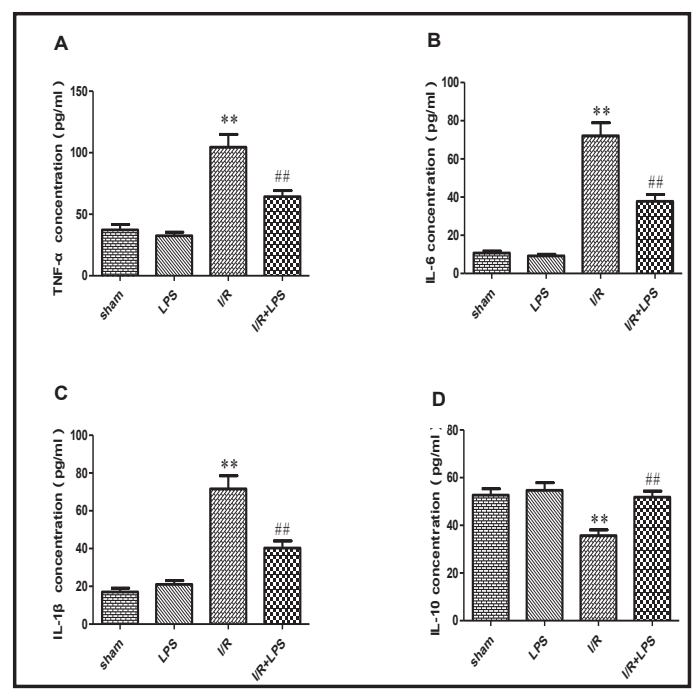

Fig. 4. ER stress-CHOP mediated signaling pathway and related apoptotic pathway inhibition by low-dose LPS pretreatment on cerebral IRI. The ER stress relative proteins GRP78 (A), CHOP (B), Cleaved Caspase-12 (C) and cleaved Caspase-3 (D) from cortical penumbra were determined by Western blotting. Representative of four experiments, ER stress-CHOP mediated inflammation-associated protein level of cleaved caspase-11 (E) was further measured by Western-assisted analysis. The relative quantities of protein to GAPDH or $\beta$-actin. Data are expressed as mean \pm SEM. $* \mathrm{P}<0.05,{ }^{* *} \mathrm{P}<0.01$ versus sham group; ${ }^{\#}<0.05,{ }^{\# \#} \mathrm{P}<0.01$ versus I/R group.

to the I/R group (TNF- $\alpha 64.33 \pm 11$ vs. $104.5 \pm 15 \mathrm{pg} / \mathrm{ml}$, IL-6 $37.83 \pm 8.3$ vs. $72.17 \pm 9 \mathrm{pg} / \mathrm{ml}, \mathrm{P}<0.05$, respectively). By contrast, the concentration of the anti-inflammatory factor IL-10 in the $\mathrm{I} / \mathrm{R}+\mathrm{LPS}$ group was clearly higher than in the I/R group (IL-10 49.6 \pm 1.1 vs. $35.8 \pm 1.2 \mathrm{pg} / \mathrm{ml}, \mathrm{P}<0.05$ ). The results showed that LPS pretreatment inhibited cerebral inflammation after IRI.

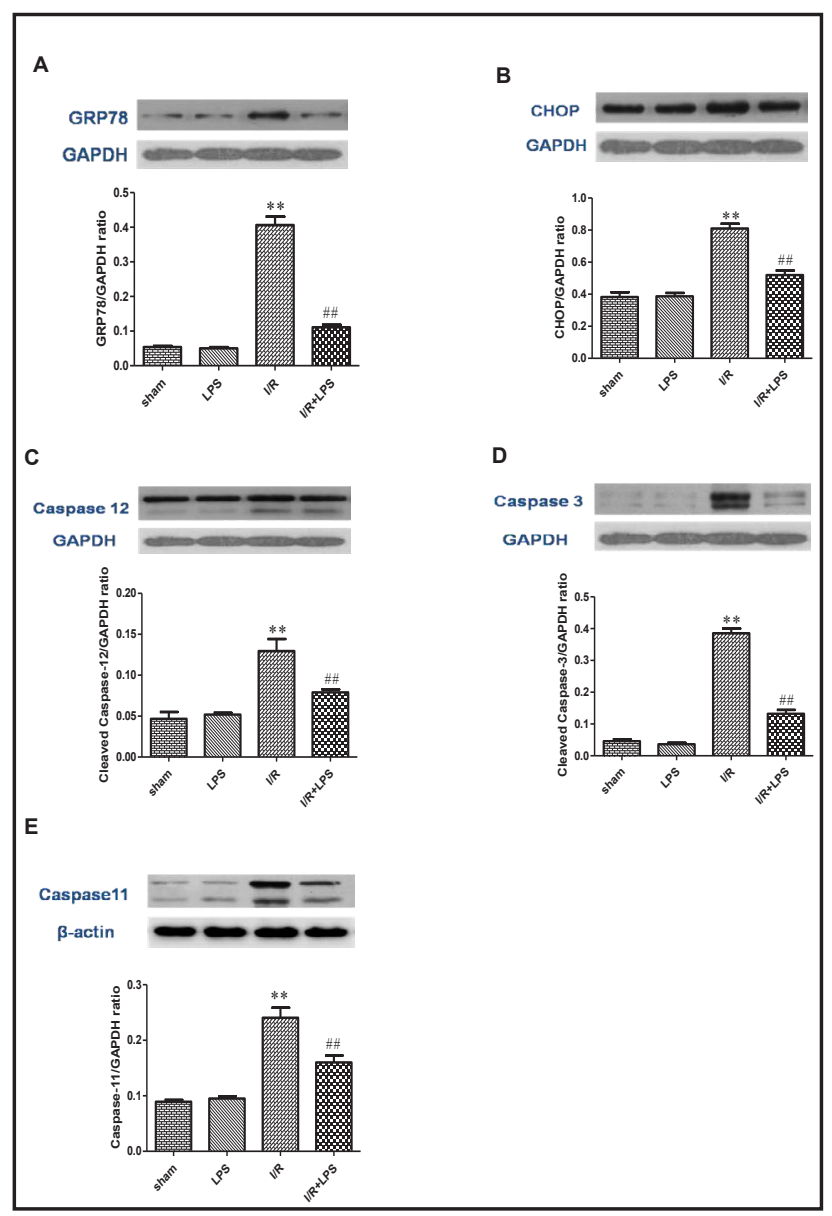

ER stress-CHOP mediated signalling pathway is involved in the protective effect of the LPS pretreatment in cerebral IRI

To assess whether ER stress-CHOP was activated upon LPS pretreatment as a neuroprotective mechanism, the expression of related proteins (GRP78, CHOP, caspase-12 and caspase-3) was further detected by western blotting (Fig. 4). According to the results, the expression of GRP78, CHOP and cleaved Caspase-12 in the I/R group was clearly increased compared to the control group $(\mathrm{P}<0.05)$, but in the LPS+I/R group, it was considerably 
decreased $(\mathrm{P}<0.05$, Figs. 4A, $B$ and $C)$. As illustrated in Fig. 5, immunohistochemistry showed that the expression of CHOP in the I/R group was significantly increased in the nucleus compared with the sham group. However, the expression of CHOP after the LPS pretreatment not only decreased significantly but also remarkably inhibited the transcription into the nucleus compared with the I/R group, which was consistent with the western blot result. To further investigate the effect of the LPS pretreatment on brain cell apoptosis, the cleaved caspase-3 activity was measured by western blot analysis (Fig. 4D). Compared with the sham group, cleaved caspase-3 protein expression was significantly increased in the I/R group. However, its protein expression was significantly decreased in the I/R+LPS group. These results indicated that the ER stress-CHOP mediated apoptosis signalling pathway was inhibited by LPS pretreatment in cerebral IRI.

The ER stress-CHOP pathway also participates in LPS-induced inflammation via inflammation-associated caspase-11 and IL-1 $\beta$. Thus, the protein level of caspase-11 was measured by a western blot analysis, and IL-1 $\beta$ was analysed by ELISA. The protein level of cleaved caspase-11 in the I/R+LPS group was obviously decreased compared with the I/R group $(\mathrm{P}<0.05$, Fig. $4 \mathrm{E})$. The concentration of IL-1 $\beta$ in the I/R+LPS group was significantly lower than that in the I/R group (Fig. 3C). These results showed that LPS pretreatment played an important role in the reduction of ER stress-CHOP related inflammatory after cerebral IRI.

\section{Discussion}

Brain injury caused by cerebral I/R is a common and serious clinical disease with a high mortality and morbidity. At present, there is no pharmacological method for the treatment of cerebral IRI, which highlights the urgent need for the development of cerebral I/R therapy strategies. However, cerebral IRI is a highly complex pathophysiological process with little understanding. The neuronal damage in the ischaemic penumbra after cerebral IRI is slow and reversible. Therefore, a reduction of the ischaemic penumbra injury is considered a key therapeutic strategy for acute cerebral IRI [2]. In the present study, we observed that LPS pretreatment alleviated cerebral IRI by inhibiting inflammation and apoptosis. This treatment's protective mechanism may be related to the ER stress-CHOP mediated signalling pathway. The treatment strategy attenuated the progression of brain injury and provided an extended treatment window for neuroprotection.

In ischaemic stroke, ischaemia and hypoxia in brain cells cause free radicals, calcium overload and an inflammatory reaction, leading to cell necrosis or apoptosis. ER stress plays an important role in this process and is thought to underlie many pathogenic progressions of the cerebral IRI [5]. Under physiological conditions, the protein activity of the ER chaperone GRP78 is inhibited. However, under pathological conditions, such as cerebral ischaemia/ hypoxia, GRP78 is released and subsequently induces the expression of ER stress genes, 
such as CHOP and caspase-12, eventually leading to apoptosis [32-34]. In this study, the protein expression levels of GRP78 and caspase-12 increased significantly after cerebral I/R, demonstrating that cerebral I/R led to ER stress.

CHOP, a transcription factor that belongs to the C/EBP transcription factor family, is a signalling event underlying ER stress-induced apoptosis. Under physiological conditions, CHOP is present in the cytosol, and its expression level is very low. However, under stress conditions, such as focal cerebral ischaemia, the expression of CHOP increases significantly and is transcribed into the nucleus $[9,35,36]$. It is demonstrated that CHOP-induced apoptosis is involved in the pathogenesis of many diseases, including cerebral ischaemia, neurodegenerative diseases and diabetes $[9,13]$. The ER stress-CHOP pathway is crucial in cerebral IRI-induced cell death [37]. In our study, cerebral I/R resulted in a significant increase in CHOP and caspase-3 protein expression and the transcription of CHOP from the cytoplasm to the nucleus. The number of apoptotic cells also increased significantly after I/R. The results proved that the ER stress-CHOP pathway was involved in cerebral IRI-induced apoptosis.

LPS, known as the antigen component of the gram-negative bacterial cell wall, causes severe sepsis and inflammation [14]. In cerebral IRI, many studies support the view that LPS aggravates cerebral IRI [38-42]. There are also reports of the neuroprotective effects of LPS pretreatment on cerebral IRI. The mechanism of neuroprotection has not been thoroughly elucidated to date [43-45]. In our study, we assessed the neuroprotective effect of LPS pretreatment against cerebral IRI by the neurological deficit score and the cerebral infarction volume. As previously published, our results showed that the neurological deficit score and infarct volume, in the mice after the LPS administration, were significantly improved, indicating that there was a neuroprotective effect on cerebral IRI.

The pathogenesis of many diseases is mediated through the ER stress-CHOP pathway, such as myocardial reperfusion injury [46], brain IRI [37], and lung diseases [19]. However, notably few studies have reported that the ER stress-CHOP mediated signalling pathway participates in the neuroprotective effect of LPS pretreatment. In our study, the expressions of GRP78, caspase-12 and CHOP were obviously inhibited by LPS pretreatment. As illustrated in Fig. 5, the immunohistochemistry showed that the expression of CHOP was not only decreased but also the transcription to the nucleus was significantly reduced after the LPS pretreatment. To assess whether the ER stress-CHOP apoptosis pathway was activated upon LPS pretreatment, as a neuroprotective mechanism, we further examined apoptosis in the brain cells and the related apoptotic protein caspase- 3 after LPS pretreatment. In our study, the number of apoptotic cells was evidently decreased, and the protein expression of caspase-3 was significantly suppressed by LPS pretreatment. Collectively, we demonstrated that the ER stress-CHOP signalling apoptosis pathway was inhibited by LPS pretreatment in cerebral IRI.

Recently, numerous studies have shown that inflammatory response plays a key role in the secondary brain injury after cerebral I/R $[47,48]$. A large number of inflammatory cytokines (TNF- $\alpha$, IL-1 $\beta$, IL-6, IL-10) are involved in brain cell apoptosis and necrosis after I/R [47-50]. Reducing the inflammatory factors could protect the brain from IRI. TNF- $\alpha$, IL$1 \beta$, and IL- 6 are the most well-known cytokines involved in the inflammatory response caused by cerebral ischaemia [51]. While IL-10 plays a key role in the prevention of inflammation and is a potent anti-inflammatory cytokine [52]. The ER stress-CHOP pathway participates in myocardial inflammation and cardiomyocyte apoptosis induced by IRI [46] and plays a key role in inflammatory-associated cysteine proteases in pancreatitis [17]. In hepatocyte IRI, LPS pretreatment markedly improved hepatocyte function in I/R-injured rats via the ER stress pathway proteins ATF4-CHOP pathway [16]. Numerous studies demonstrate that the ER-stress CHOP pathway was also involved in LPS-induced inflammation by inducing the inflammation associated with caspase 11 and IL-1 $\beta[15,17,18]$. These studies suggest that the ER stress-CHOP pathway is very important in the pathogenesis of inflammatory diseases. However, little is known regarding the ER stress-CHOP signalling pathway as it relates to the protective effects of LPS pretreatment. CHOP, as an IL-1 $\beta$ maturation process activator, is 
Ly et al.: LPS Pretreatment and Cerebral Ischaemia/Reperfusion Injury

highly important in the pathogenesis of inflammation, serving to induce caspase-11 [18]. Similarly, in the same article, the ER stress-CHOP pathway was shown to participate in the process of LPS-induced activation of caspase-11 and IL-1 $1 \beta$. Consistent with these results, our study found that the concentrations of pro-inflammatory cytokines (TNF- $\alpha$, IL-6, IL-1 $\beta$ ) were significantly decreased after LPS pretreatment, while the concentration of the antiinflammatory factor IL-10 was significantly increased. Similarly, the expression level of caspase-11 protein was significantly increased. Our results showed that LPS pretreatment played an important role in reducing ER stress-CHOP- related inflammatory responses after cerebral IRI.

\section{Conclusion}

Our report demonstrates that LPS pretreatment attenuates cerebral IRI by inhibiting inflammation and apoptosis, and the mechanism of cerebral protection may be via the ER stress-CHOP mediated signalling pathway. Severe cerebral IRI may be disabling, even fatal, and the ER stress-CHOP mediated signalling pathway could be a potential target for developing new neuroprotective strategies against cerebral IRI.

\section{Acknowledgements}

Funding: Mingqing Peng was supported by the Natural Science Fund Projects of Chongqing Yongchuan Science and Technology Commission (2015nc5023); Chong Liu was supported by the "PhD and Master's Funding" of Tianjin Fourth Central Hospital, China (2017S05); Fang Zheng was supported by Natural Science Foundation of China (81102543) and Tianjin Natural Science Foundation (17JCYBJC26500).

\section{Disclosure Statement}

The authors declare no conflicts of interest.

\section{References}

1 Lipton P: Ischemic cell death in brain neurons. Physiol Rev 1999;79:1431-1568.

-2 Heiss WD: The ischemic penumbra: correlates in imaging and implications for treatment of ischemic stroke. The Johann Jacob Wepfer award 2011. Cerebrovasc Dis 2011;32:307-320.

- Z Zaidat O0, Lazzaro MA, Liebeskind DS, Janjua N, Wechsler L, Nogueira RG, Edgell RC, Kalia JS, Badruddin A, English J, Yavagal D, Kirmani JF, Alexandrov AV, Khatri P: Revascularization grading in endovascular acute ischemic stroke therapy. Neurology 2012;79:S110-116.

-4 Lehotsky J, Racay P, Pavlikova M, Tatarkova Z, Urban P, Chomova M, Kovalska M, Kaplan P: Cross-talk of intracellular calcium stores in the response to neuronal ischemia and ischemic tolerance. Gen Physiol Biophys 2009;28 Spec No Focus:F104-114.

5 Xin Q, Ji B, Cheng B, Wang C, Liu H, Chen X, Chen J, Bai B: Endoplasmic reticulum stress in cerebral ischemia. Neurochem Int 2014;68:18-27.

6 Kozutsumi Y, Segal M, Normington K, Gething MJ, Sambrook J: The presence of malfolded proteins in the endoplasmic reticulum signals the induction of glucose-regulated proteins. Nature 1988;332:462-464.

7 Hosoi T, Ozawa K: Endoplasmic reticulum stress in disease: mechanisms and therapeutic opportunities. Clin Sci (Lond) 2009;118:19-29.

-8 Gardner BM, Pincus D, Gotthardt K, Gallagher CM, Walter P: Endoplasmic reticulum stress sensing in the unfolded protein response. Cold Spring Harb Perspect Biol 2013;5:a013169. 


\section{Cellular Physiology Cell Physiol Biochem 2018;45:2246-2256 \begin{tabular}{l|l} 
and Biochemistry Publishe.1159/000488170 & $\begin{array}{l}\text { (c) } 2018 \text { The Author(s). Published by S. Karger AG, Basel } \\
\text { www.karger.com/cpb }\end{array}$
\end{tabular}

Oyadomari S, Mori M: Roles of CHOP/GADD153 in endoplasmic reticulum stress. Cell Death Differ 2004;11:381-389.

10 Hitomi J, Katayama T, Taniguchi M, Honda A, Imaizumi K, Tohyama M: Apoptosis induced by endoplasmic reticulum stress depends on activation of caspase-3 via caspase-12. Neurosci Lett 2004;357:127-130.

$\checkmark 11$ Rao RV, Ellerby HM, Bredesen DE: Coupling endoplasmic reticulum stress to the cell death program. Cell Death Differ 2004;11:372-380.

-12 Nagai H, Noguchi T, Takeda K, Ichijo H: Pathophysiological roles of ASK1-MAP kinase signaling pathways. J Biochem Mol Biol 2007;40:1-6.

13 Yang Y, Liu L, Naik I, Braunstein Z, Zhong J, Ren B: Transcription Factor C/EBP Homologous Protein in Health and Diseases. Front Immunol 2017;8:1612.

14 Rosadini CV, Kagan JC: Early innate immune responses to bacterial LPS. Curr Opin Immunol 2017;44:14-19.

15 Nakayama Y, Endo M, Tsukano H, Mori M, Oike Y, Gotoh T: Molecular mechanisms of the LPS-induced nonapoptotic ER stress-CHOP pathway. J Biochem 2010;147:471-483.

16 Rao J, Qin J, Qian X, Lu L, Wang P, Wu Z, Zhai Y, Zhang F, Li G, Wang X: Lipopolysaccharide preconditioning protects hepatocytes from ischemia/reperfusion injury (IRI) through inhibiting ATF4-CHOP pathway in mice. PLoS One 2013;8:e65568.

17 Suyama K, Ohmuraya M, Hirota M, Ozaki N, Ida S, Endo M, Araki K, Gotoh T, Baba H, Yamamura K: C/EBP homologous protein is crucial for the acceleration of experimental pancreatitis. Biochem Biophys Res Commun 2008;367:176-182.

18 Endo M, Mori M, Akira S, Gotoh T: C/EBP homologous protein (CHOP) is crucial for the induction of caspase-11 and the pathogenesis of lipopolysaccharide-induced inflammation. J Immunol 2006;176:62456253.

19 Endo M, Oyadomari S, Suga M, Mori M, Gotoh T: The ER stress pathway involving CHOP is activated in the lungs of LPS-treated mice. J Biochem 2005;138:501-507.

-20 Thornberry NA, Bull HG, Calaycay JR, Chapman KT, Howard AD, Kostura MJ, Miller DK, Molineaux SM, Weidner JR, Aunins J, et al.: A novel heterodimeric cysteine protease is required for interleukin-1 beta processing in monocytes. Nature 1992;356:768-774.

-21 Toyoda T, Kassell NF, Lee KS: Induction of tolerance against ischemia/reperfusion injury in the rat brain by preconditioning with the endotoxin analog diphosphoryl lipid A. J Neurosurg 2000;92:435-441.

-22 Chang R, Wang Y, Chang J, Wen L, Jiang Z, Yang T, Yu K: LPS preconditioning ameliorates intestinal injury in a rat model of hemorrhagic shock. Inflamm Res 2014;63:675-682.

23 Yao YW, Zhang GH, Zhang YY, Li WD, Wang CH, Yin CY, Zhang FM: Lipopolysaccharide pretreatment protects against ischemia/reperfusion injury via increase of HSP70 and inhibition of NF-kappaB. Cell Stress Chaperones 2011;16:287-296.

-24 Longa EZ, Weinstein PR, Carlson S, Cummins R: Reversible middle cerebral artery occlusion without craniectomy in rats. Stroke 1989;20:84-91.

-25 Swanson RA, Morton MT, Tsao-Wu G, Savalos RA, Davidson C, Sharp FR: A semiautomated method for measuring brain infarct volume. J Cereb Blood Flow Metab 1990;10:290-293.

-26 Ashwal S, Tone B, Tian HR, Cole DJ, Pearce WJ: Core and penumbral nitric oxide synthase activity during cerebral ischemia and reperfusion. Stroke 1998;29:1037-1046; discussion 1047.

-27 Huang H, Chen YM, Zhu F, Tang ST, Xiao JD, Li LL, Lin XJ: Down-regulated Na(+)/K(+)-ATPase activity in ischemic penumbra after focal cerebral ischemia/reperfusion in rats. Int J Clin Exp Pathol 2015;8:1270812717.

28 Collaco-Moraes Y, Aspey BS, de Belleroche JS, Harrison MJ: Focal ischemia causes an extensive induction of immediate early genes that are sensitive to MK-801. Stroke 1994;25:1855-1860; discussion 1861.

29 Muller TB, Haraldseth 0, Unsgard G: Characterization of the microcirculation during ischemia and reperfusion in the penumbra of a rat model of temporary middle cerebral artery occlusion: a laser Doppler flowmetry study. Int J Microcirc Clin Exp 1994;14:289-295.

-30 Takagi K, Ginsberg MD, Globus MY, Dietrich WD, Martinez E, Kraydieh S, Busto R: Changes in amino acid neurotransmitters and cerebral blood flow in the ischemic penumbral region following middle cerebral artery occlusion in the rat: correlation with histopathology. J Cereb Blood Flow Metab 1993;13:575-585.

-31 Memezawa H, Minamisawa H, Smith ML, Siesjo BK: Ischemic penumbra in a model of reversible middle cerebral artery occlusion in the rat. Exp Brain Res 1992;89:67-78. 


\section{Cellular Physiology Cell Physiol Biochem 2018;45:2246-2256 \begin{tabular}{ll|l} 
aOI: 10.1159/000488170 & $\begin{array}{l}\text { O 2018 The Author(s). Published by S. Karger AG, Basel } \\
\text { www.karger.com/cpb }\end{array}$ \\
\hline
\end{tabular}}

-32 Gorman AM, Healy SJ, Jager R, Samali A: Stress management at the ER: regulators of ER stress-induced apoptosis. Pharmacol Ther 2012;134:306-316.

33 Garcia de la Cadena S, Massieu L: Caspases and their role in inflammation and ischemic neuronal death. Focus on caspase-12. Apoptosis 2016;21:763-777.

-34 Paschen W, Mengesdorf T: Endoplasmic reticulum stress response and neurodegeneration. Cell Calcium 2005;38:409-415.

-35 Ye Z, Wang N, Xia P, Wang E, Liao J, Guo Q: Parecoxib suppresses CHOP and Foxo1 nuclear translocation, but increases GRP78 levels in a rat model of focal ischemia. Neurochem Res 2013;38:686-693.

-36 Ron D, Habener JF: CHOP, a novel developmentally regulated nuclear protein that dimerizes with transcription factors C/EBP and LAP and functions as a dominant-negative inhibitor of gene transcription. Genes Dev 1992;6:439-453.

-37 Tajiri S, Oyadomari S, Yano S, Morioka M, Gotoh T, Hamada JI, Ushio Y, Mori M: Ischemia-induced neuronal cell death is mediated by the endoplasmic reticulum stress pathway involving CHOP. Cell Death Differ 2004;11:403-415.

-38 Liu X, Chen X, Zhu Y, Wang K, Wang Y: Effect of magnolol on cerebral injury and blood brain barrier dysfunction induced by ischemia-reperfusion in vivo and in vitro. Metab Brain Dis 2017;10.1007/s11011017-0004-6

39 Zhang P, Guo ZF, Xu YM, Li YS, Song JG: N-Butylphthalide (NBP) ameliorated cerebral ischemia reperfusioninduced brain injury via HGF-regulated TLR4/NF-kappaB signaling pathway. Biomed Pharmacother 2016;83:658-666.

40 Zhuang P, Wan Y, Geng S, He Y, Feng B, Ye Z, Zhou D, Li D, Wei H, Li H, Zhang Y, Ju A: Salvianolic Acids for Injection (SAFI) suppresses inflammatory responses in activated microglia to attenuate brain damage in focal cerebral ischemia. J Ethnopharmacol 2017;198:194-204.

-41 Anthony Jalin AM, Lee JC, Cho GS, Kim C, Ju C, Pahk K, Song HY, Kim WK: Simvastatin Reduces Lipopolysaccharides-Accelerated Cerebral Ischemic Injury via Inhibition of Nuclear Factor-kappa B Activity. Biomol Ther (Seoul) 2015;23:531-538.

42 Ji B, Han Y, Liu Q, Liu X, Yang F, Zhou R, Lian Q Cao H, Li J: [Curcumin improves the impaired working memory in cerebral ischemia-reperfusion rats by inhibiting proinflammatory cytokines]. Zhonghua Yi Xue Za Zhi 2014;94:1029-1033.

43 Vartanian KB, Stevens SL, Marsh BJ, Williams-Karnesky R, Lessov NS, Stenzel-Poore MP: LPS preconditioning redirects TLR signaling following stroke: TRIF-IRF3 plays a seminal role in mediating tolerance to ischemic injury. J Neuroinflammation 2011;8:140.

44 Hickey E, Shi H, Van Arsdell G, Askalan R: Lipopolysaccharide-induced preconditioning against ischemic injury is associated with changes in toll-like receptor 4 expression in the rat developing brain. Pediatr Res 2011;70:10-14.

45 Wang TH, Xiong LL, Yang SF, You C, Xia QJ, Xu Y, Zhang P, Wang SF, Liu J: LPS Pretreatment Provides Neuroprotective Roles in Rats with Subarachnoid Hemorrhage by Downregulating MMP9 and Caspase3 Associated with TLR4 Signaling Activation. Mol Neurobiol 2016;10.1007/s12035-016-0259-7

-46 Miyazaki Y, Kaikita K, Endo M, Horio E, Miura M, Tsujita K, Hokimoto S, Yamamuro M, Iwawaki T, Gotoh T, Ogawa H, Oike Y: C/EBP homologous protein deficiency attenuates myocardial reperfusion injury by inhibiting myocardial apoptosis and inflammation. Arterioscler Thromb Vasc Biol 2011;31:1124-1132.

-47 Sieber MW, Claus RA, Witte OW, Frahm C: Attenuated inflammatory response in aged mice brains following stroke. PLoS One 2011;6:e26288.

48 Dziedzic T: Systemic inflammation as a therapeutic target in acute ischemic stroke. Expert Rev Neurother 2015;15:523-531.

49 Bonaventura A, Liberale L, Vecchie A, Casula M, Carbone F, Dallegri F, Montecucco F: Update on Inflammatory Biomarkers and Treatments in Ischemic Stroke. Int J Mol Sci 2016;17:

50 Xing J, Lu J: HIF-1alpha Activation Attenuates IL-6 and TNF-alpha Pathways in Hippocampus of Rats Following Transient Global Ischemia. Cell Physiol Biochem 2016;39:511-520.

-51 Ziebell JM, Morganti-Kossmann MC: Involvement of pro- and anti-inflammatory cytokines and chemokines in the pathophysiology of traumatic brain injury. Neurotherapeutics 2010;7:22-30.

52 Ouyang W, Rutz S, Crellin NK, Valdez PA, Hymowitz SG: Regulation and functions of the IL-10 family of cytokines in inflammation and disease. Annu Rev Immunol 2011;29:71-109. 